\title{
AN INTRODUCTION TO THE GLOBAL STRATEGY FOR PLANT CONSERVATION
}

\author{
DAVID RAE
}

The background to, and development of, the Global Strategy for Plant Conservation is described and the agreed global targets for 2010 are listed. The 16 targets are arranged in five groups as follows: understanding and documenting plant diversity, conserving plant diversity, using plant diversity sustainability, promoting education and awareness about plant diversity and building capacity for the conservation of plant diversity.

\section{BACKGROUND}

The Global Strategy for Plant Conservation arose from a resolution from the International Botanic Congress (IBC), held in St Louis, Missouri in August 1999, which called for plant conservation to be recognised as a global priority in biodiversity conservation. After the IBC a number of interested parties met in Gran Canaria in April 2000 during a meeting organised by Botanic Gardens Conservation International (BGCI) to give further consideration to this issue and consider what further steps were possible or necessary. The outcomes of their deliberations were published as the Gran Canaria Declaration (http://www.biodiv.org/doc/meetings/ cop/cop-05/information/cop-05-inf-32-en.pdf), and called for the development of a Global Strategy for Plant Conservation within the framework of the Convention on Biological Diversity (CBD).

\section{Conference of the Parties and Subsidiary Body on Scientific, Technical and Technological Advice}

Thereafter, the proposals were presented at the CBD's Conference of the Parties (COP) meeting in Nairobi, and endorsed as its Resolution V/10. This resolution requested the CBD's Subsidiary Body on Scientific, Technical and Technological Advice (SBSTTA) to give further consideration to the matter and to make specific recommendations at the next COP meeting, in 2002.

The Nairobi COP also called on the CBD Secretariat to arrange meetings with interested bodies to discuss the development of this global initiative in time for the SBSTTA meeting and as a result two informal consultations were held, both with 
Gran Canaria Group ${ }^{1}$. The first meeting took place in Montreal and the second, in May 2001, in London, organised by BGCI. At these meetings it was agreed that specific targets needed to be developed and adopted for inclusion in the Strategy, if it was going to have a significant impact. This was also additionally significant because, to date, few outcome targets have been prepared to measure the work of the CBD. Through these meetings 14 'outcome-orientated targets' were developed, aimed at:

(a) Understanding and documienting plant diversity

(b) Conserving plant diversity

(c) Using plant diversity sustainably

(d) Promoting education and awareness about plant diversity; and

(e) Building capacity for the conservation of plant diversity.

Following the May meeting in London a workshop was held at the International Association of Botanic Gardens (IABG) Congress in Cordoba which further considered the 14 targets. Other comments and refinements were added by a range of international organisations (mainly those involved in the Gran Canaria Group) as well as by the CBD authorities in several countries. In September 2001 a paper was finalised for consideration at the 7th SBSTTA meeting held in November 2001 in Montreal. At SBSTTA this draft was debated comprehensively both during the working sessions of the conference as well as at informal discussion groups. The result was a Recommendation (titled VII/8 Global strategy for plant conservation) and the targets were revised to include an additional two more, making a total of 16 .

The next stage involved working towards the next COP meeting due to be held in the Netherlands in April 2002. The recommendation from SBSTTA recognised that further work on the Strategy was necessary and work involving both national $\mathrm{CBD}$ authorities and the Gran Canaria Group continued during the months that followed. During that time the targets were further refined, as was the basis on which they had been developed. It was recognised by those active in promoting the Strategy that it was important also that national delegations were fully briefed about the importance and significance of the Strategy so that they could be urged to support it at the COP meeting.

The Strategy was duly discussed at the COP in the Netherlands and the Strategy along with the 16 targets were fully adopted. The targets provide a framework for policy formulation and a basis for monitoring. National targets developed within this framework may vary from country to country according to national priorities and capacities taking into account differences in plant diversity.

\footnotetext{
${ }^{1}$ The Gran Canaria Group is an informal grouping of representatives of a range of international and regional organisations and other major institutions involved in plant conservation, such as IUCN, WWF, IPGRI, FAO, BGCI, WCMC, IABG, the Smithsonian Institution, the Commonwealth Secretariat, the Royal Botanic Gardens of Edinburgh and Kew, Planta Europa and network organisations in Canada, the U.S.A., Australia, Africa, South East Asia and other regions. A secretariat for the Gran Canaria Group is provided by Botanic Gardens Conservation International (BGCI).
} 


\section{TARGETS}

Agreed global targets for the year 2010 are as follows:

Understanding and documenting plant diversity:

1. A widely accessible working list of known plant species, as a step towards a complete world flora.

2. A preliminary assessment of the conservation status of all known plant species, at national, regional and international level.

3. Development of models with protocols for plant conservation and sustainable use, based on research and practical experience.

Conserving plant diversity:

4. At least $10 \%$ of each of the world's ecological regions effectively conserved.

5. Protection of $50 \%$ of the most important areas for plant diversity assured.

6. At least $30 \%$ of production lands managed consistent with the conservation of plant diversity.

7. $60 \%$ of the world's threatened species conserved in situ.

8. $60 \%$ of threatened plant species in accessible ex situ collections, preferably in the country of origin, and $10 \%$ of them included in recovery and restoration programmes.

9. $70 \%$ of the genetic diversity of crops and other major socio-economically valuable plant species conserved, and associated local and indigenous knowledge maintained.

10. Management plans in place for at least 100 major alien species that threaten plants, plant communities and associated habitats and ecosystems.

Using plant diversity sustainably:

11. No species of wild flora endangered by international trade.

12. $30 \%$ of plant-based products derived from sources that are sustainably managed.

13. The decline of plant resources, and associated local and indigenous knowledge, innovations and practices, that support sustainable livelihoods, local food security and health care, halted.

Promoting education and awareness about plant diversity:

14. The importance of plant diversity and the need for its conservation incorporated into communication, education and public awareness programmes.

Building capacity for the conservation of plant diversity:

15. The number of trained people working with appropriate facilities in plant conservation increased according to national needs, to achieve the targets of this strategy. 
16. Networks for plant conservation activities established or strengthened at national, regional, and international levels.

These targets provide a framework for policy formulation and a basis for monitoring. National targets developed within this framework may vary from country to country, according to national priorities and capacities taking into account differences in plant diversity.

\section{Next Steps}

Each region and country now needs to consider how it will meet these targets and how progress will be monitored. At an international level too, urgent consideration needs to be given to the targets and how to implement them across national boundaries. In the UK, for instance, a meeting with all relevant parties was held on 5 February 2003 to consider how each of the targets might be approached. For each of the targets an invited speaker gave an explanation of the target, presented the background and baseline for the UK, described existing UK obligations and initiatives and then suggested proposed action to meet the target by 2010 .

The Strategy provides both a challenge and an opportunity for botanic gardens around the world - it is a challenge we must rise to. 